\title{
MORFOLOGIA EXTERNA DE ESPÉCIMES ADULTOS DE Paratanaisia bragai (SANTOS, 1934) (DIGENEA: EUCOTYLIDAE)
}

\author{
SOLANGE V.P.B. BRANDOLINI ${ }^{1}$; SUZANA B. AMATO²
}

\begin{abstract}
BRANDOLINI, S.V.P.B.; AMATO, S.B. [External morphology of the adults specimens of Paratanaisia bragai (Santos, 1934)(Diginea; Eucotylidae).] Morfologia externa de espécimes adultos de Paratanaisia bragai (Santos, 1934) (Digenea: Eucotylidae). Revista Brasileira de Parasitologia Veterinária, v. 16, n. 3, p. 129-132, 2007. Departamento de Biologia Animal, Instituto de Biologia, Universidade Federal Rural do Rio de Janeiro. Caixa postal 74555, Seropédica, RJ 23851-970. E-mail: solangeb@ufrrj.br

Adult specimens of Paratanaisia bragai collected from renal ducts of Columba livia naturally infected, were fixed in glutaraldehyde $2.5 \%$ and processed for scanning electron microscopy. Two distinct types of scales had been identified, bifid and simple scales, disposed in transversal rows, on the tegument of dorsal and ventral face of the body. Also show in details the oral sucker subterminal, well developed and acetabulum of size reduced, located in third medium of the body, surrounded for small protuberance and four papillae on its edge.
\end{abstract}

KEY WORDS: Paratanaisia bragai, tegument, scales, acetabulum.

\section{RESUMO}

Espécimes adultos de Paratanaisia bragai foram coletados de ductos renais de Columba livia infectados naturalmente, fixados em glutaraldeído 2,5\% e processados para microscopia eletrônica de varredura. Foram identificados dois tipos distintos de escamas, escamas bífidas e simples, dispostas em fileiras transversais no tegumento das faces dorsal e ventral do corpo. Também mostrando em detalhes a ventosa oral subterminal, bem desenvolvida e acetábulo de tamanho reduzido, localizado no terço médio do corpo, circundado por pequenas protuberâncias e quatro papilas dispostas regularmente na sua margem.

PALAVRAS-CHAVE: Paratanaisia bragai, tegumento, escamas, acetábulo.

\section{INTRODUÇÃO}

A mais recente revisão da família Eucotylidae foi feita por Kanev et al. (2002). De acordo com estes autores, Eucotylidae inclui duas subfamílias: Eucotylinae Skrjabin, 1924 que tem como principal característica diagnóstica a presença de um

\footnotetext{
${ }^{1}$ Departamento de Biologia Animal, Instituto de Biologia, Universidade Federal Rural do Rio de Janeiro. Caixa postal 74555, Seropédica, RJ 23851-970. E-mail: solangeb@ufrrj.br

${ }^{2}$ Departamento de Zoologia. Universidade Federal do Rio Grande do Sul, Caixa postal 15014, Porto Alegre, RS 91501-970. E-mail: sbamato@ufrgs.br
}

colar muscular em forma cônica ou triangular delimitando a região anterior do resto do corpo e Tanaisiinae Freitas, 1951 (sinônimo de Tanaisiinae Yamaguti,1958) que não apresenta este colar muscular anterior. Em Tanaisiinae foram alocados os gêneros Tanaisia (espécie tipo é T. fedtschenkoi Skrjabin, 1924), Paratanaisia com espécie tipo P. bragai (Santos, 1934) Freitas, 1959 e Tamerlania com espécie tipo T. zarudnyi Skrjabin, 1924.

Espécimes adultos de Paratanaisia bragai são encontrados em ductos renais de diversas espécies de aves domésticas e silvestres (SANTOS, 1934; BYRD; DENTON, 1950; FREITAS, 1951; ARNIZAUT et al., 1992; FEDYNICH et al., 1996; GOMES et al., 2005).

Santos (1934) ao descrever Tamerlanea bragai, induziu a um erro na grafia do nome genérico, Tamerlanea e não Tamerlania como proposto por Skrjabin (1924), sendo este erro repetido por outros autores. Santos (1934) classificou este digenético como monóstome pela ausência do acetábulo. Posteriormente, trabalhos realizados por Maldonado e Hoffman (1941) e Maldonado (1945) demonstraram a presença do acetábulo na cercária e em espécimes jovens de Paratanaisia bragai (referidos como Tanaisia bragai). Segundo Yamaguti (1958) um pequeno acetábulo poderia estar ocasionalmente presente. Byrd e Denton (1950) quando transferiram a espécie descrita por Santos (1934) para o gênero Tanaisia destacaram na descrição que se tratava de um trematódeo dístome de tamanho pequeno a médio. Entretanto, não observaram o acetábulo no material examinado. Se- 
gundo Freitas (1951), as escamas e espinhos presentes no tegumento dos eucotilídeos deveriam ser considerados como caracter distintivo de grande importância para a sistemática da família. Posteriormente, Odening (1963) afirmou ser necessária uma análise minuciosa de escamas e espinhos das espécies de Eucotylidae, uma vez que a maioria dos trabalhos realizados, até então, não forneciam uma descrição detalhada e muitas vezes não faziam sequer uma distinção exata entre escama e espinho. Este último autor considerou a família Eucotylidae como homogênea, entre outros aspectos pela presença de escamas, tipo crista, tanto em Tanaisiinae quanto em Eucotylinae.

Em função das informações disponíveis na literatura serem algumas vezes incompletas e/ou conflitantes, este presente estudo teve por objetivos demonstrar e descrever, com auxílio da microscopia eletrônica de varredura, o acetábulo e as escamas presentes no tegumento de espécimes adultos de P. bragai.

\section{MATERIAL E MÉTODOS}

Onze espécimes adultos de pombos da espécie Columba livia Gmelin, procedentes do bairro Campo Lindo, Município de Seropédica, RJ, foram submetidos a exames de fezes pelo método de sedimentação, segundo De Carli (1994). Sete apresentaram-se infectados por $P$. bragai, o que corresponde a 63,6\% de prevalência de infecção e foram eutanasiados e necropsiados conforme os princípios éticos estabelecidos pela Comissão de Ética em Pesquisa e Experimentação em Biologia - CEPEB/UFRRJ.

Espécimes adultos de $P$. bragai foram coletados dos ductos renais de pombos, naturalmente infectados, e fixados sem compressão em glutaraldeído 2,5\% em tampão fosfato em $\mathrm{pH}$ 7,2 por 2 horas, seguido de três lavagens em tampão fosfato.

As amostras foram desidratadas em álcool etílico em concentrações crescentes até etanol absoluto; seguindo-se a desidratação em série cetônica, durante 15 minutos em cada concentração e a determinação do ponto crítico, com dessecação completa das amostras com $\mathrm{CO}_{2}$ (BANCROFT; STEVENS, 1996). Os espécimes foram montados em suportes de alumínio e metalizados com ouro por 60 segundos. Sendo, posteriormente, fotomicrografados ao microscópio eletrônico de varredura Stereoscan 200, com câmera Nikon F-301 acoplada e filme Neopan SS Iso 100 da Fuji.

As medidas dos espécimes correspondem ao valor médio para 10 espécimes, estão seguidas pela amplitude de variação e são expressas em $\mu \mathrm{m}$. Para as escamas bífidas são apresentadas as medidas do comprimento e da largura e para as escamas simples somente o comprimento, em função da sua forma cônica.

\section{RESULTADOS E DISCUSSÃO}

A espécie $P$. bragai apresenta corpo alongado, achatado dorso-ventralmente, com comprimento de 1735,14 $\mu \mathrm{m}$ (1674,8 a $1756,34 \mu \mathrm{m})$, a ventosa oral desenvolvida e o acetábulo de tamanho reduzido, conforme pode ser observado na Figura 1. A ventosa oral com 133,47 $\mu \mathrm{m}(128,44$ a $140,07 \mu \mathrm{m})$ e $102,07 \mu \mathrm{m}(98,61$ a 103,34 $\mu \mathrm{m})$ para o maior e menor diâmetro, respectivamente, apresenta uma série de papilas dispostas irregularmente ao seu redor (Figura 2). O acetábulo apresenta $40,83 \mu \mathrm{m}(37,70$ a $43,96 \mu \mathrm{m})$ e $20,41 \mu \mathrm{m}(18,30$ a $22,52 \mu \mathrm{m})$ para o maior e menor diâmetro, respectivamente e, está localizado no terço médio do corpo, circundado por pequenas protuberâncias e quatro papilas dispostas regularmente em seu bordo (Figuras 3 e 4).

A presença do acetábulo na cercária e em espécimes jovens de Tanaisia bragai (= P. bragai) foi registrada por Maldonado e Hoffman (1941) e por Maldonado (1945). Segundo esses autores, com o desenvolvimento do parasito, o acetábulo poderia crescer ligeiramente, mas eventualmente atrofiava e desaparecia após o parasito ter atingido a maturidade sexual. Sendo, portanto, considerado como uma estrutura vestigial. Entretanto, as características morfológicas do acetábulo observado nos espécimes adultos de $P$. bragai demonstram que esta estrutura, embora de tamanho reduzido, é funcional.

O acetábulo não foi observado nos espécimes examinados por Byrd e Denton (1950) quando estes autores transferiram a espécie descrita pos Santos (1934) para o gênero Tanaisia. Embora, estes autores tenham relatado que se tratava de um trematódeo dístome de tamanho pequeno a médio. Segundo Yamaguti (1958) um pequeno acetábulo poderia estar ocasionalmente presente. Keller e Araújo (1992) em uma breve descrição do espécime adulto da espécie $P$. bragai não mencionaram o acetábulo. Dessa forma, o presente, estudo ratifica a presença do acetábulo em espécimes adultos de $P$. bragai.

O tegumento, de textura irregular, apresenta-se bastante enrugado, com uma série de escamas, dispostas em fileiras transversais, nas faces dorsal e ventral do corpo, sempre voltadas para a extremidade posterior do corpo, estando mais concentradas no terço anterior da face ventral do corpo (Figuras 4 - 6). As escamas são achatadas e de dois tipos distintos, um que apresenta a lâmina dividida em dois elementos, com as extremidades livres, sendo nesse caso chamada de escama bífida, e outro que não apresenta essa divisão e é chamada de escama simples (Figuras 5 e 6). A escama simples apresenta $1,07 \mu \mathrm{m}(0,92$ a $1,57 \mu \mathrm{m})$ de comprimento e a esca-

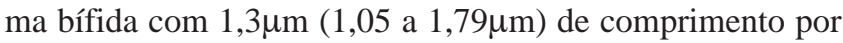
$1,2 \mu \mathrm{m}(1,08$ a $1,37 \mu \mathrm{m})$ de largura. As medidas das escamas, dos espécimes do presente estudo, enquadram-se nas amplitudes de variação apresentadas por Stunkard (1945) e Freitas (1951), fazendo-se as respectivas correspondência de unidades. Contudo, esses autores não registraram a presença de dois tipos distintos de escamas.

Santos (1934), Tubangui e Masilungan (1941) e Byrd e Denton (1950) entre outros autores relatam a presença de estruturas semelhantes a escamas ou espinhos no tegumento de $P$. bragai sem, contudo fazerem uma distinção clara entre estes dois tipos de estruturas. Stunkard (1945) fez a primeira 

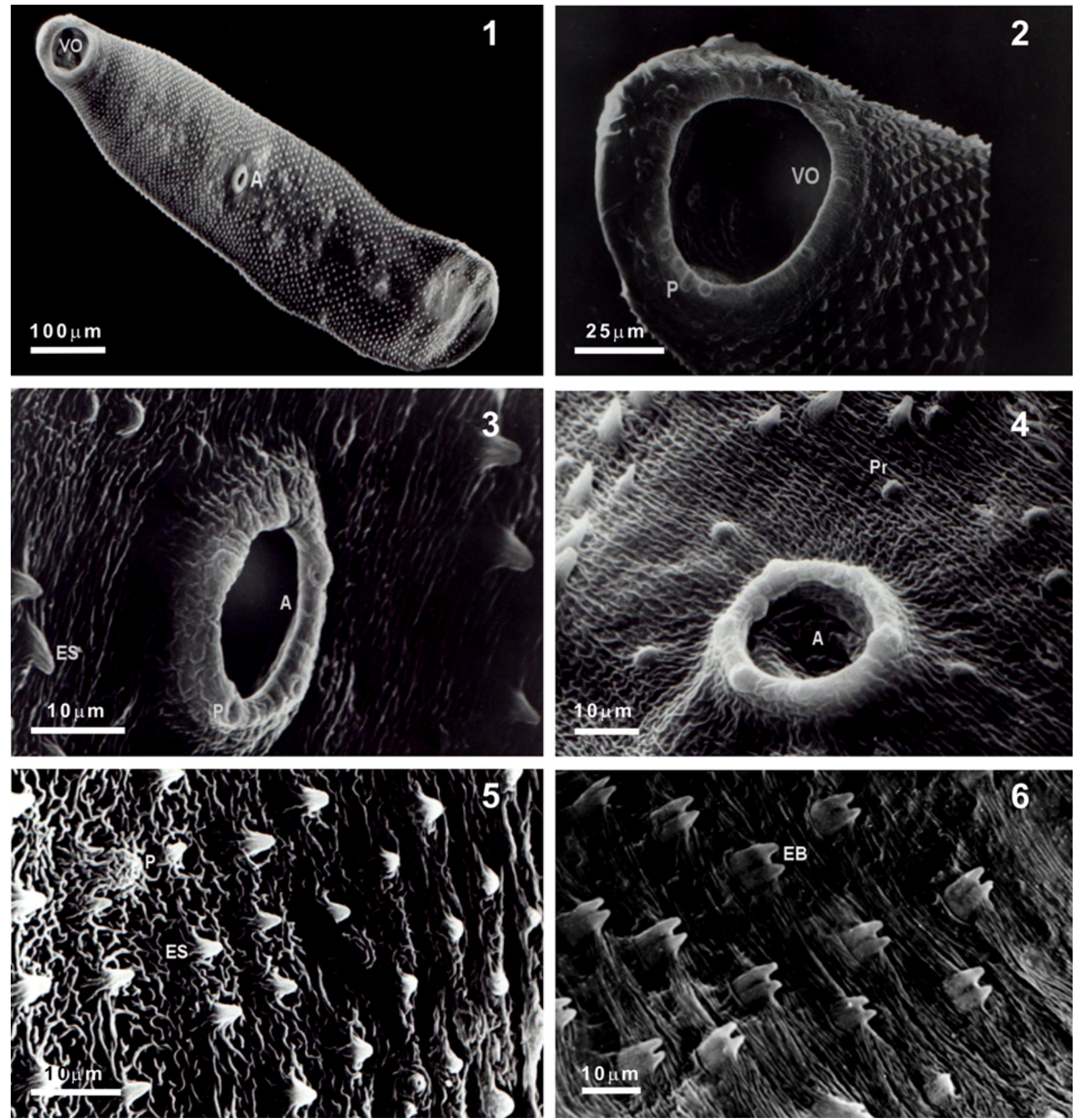

Figuras 1-6. Espécime adulto de Paratanaisia bragai: 1. Aspecto geral da superfície ventral do corpo, ventosa oral (VO) e acetábulo (A); 2. Extremidade anterior, mostrando a ventosa oral com papilas $(P)$ dispostas irregularmente ao seu redor; 3 . Acetábulo, com detalhe para as papilas e escamas simples (ES); 4. Pequenas protuberâncias ( $\mathrm{Pr}$ ) no tegumento de textura irregular ao redor do acetábulo; 5 . Superfície dorsal do corpo mostrando o tegumento de textura irregular, com escamas simples e papilas; 6 . Vista ventral do corpo evidenciando as escamas bífidas (EB), dispostas em fileiras transversais.

descrição mais detalhada das escamas presentes no tegumento de $T$. bragai (= P. bragai), a partir de material coletado em Porto Rico e usou a denominação “escama, tipo crista”. Na Europa, Dollfus (1946) descreveu escamas semelhantes em cortes de . gallica (= T. zarudnyi). Em função das semelhanças encontradas entre as escamas de várias espécies, Freitas (1951) considerou as escamas e espinhos do tegumento dos eucotilídeos como um caracter distintivo de grande importância para a sistemática da família. Com os resultados obtidos, no presente estudo, ratifica-se a presença de escamas bífidas
(= tipo crista) no tegumento de espécimes adultos de $P$. bragai e apresenta-se um segundo tipo de escama, escama simples.

\section{REFERÊNCIAS BIBLIOGRÁFICAS}

ARNIZAUT, A.B.; HAYES, L.; OLSEN, G.H.; TORRES, J.S.; RUIZ, C.; PÉREZ-RIVERA, R. An epizootic of Tanaisia bragai in a captive population of Puerto Rican plain pigeon (Columba inornata wetmorei). Annals New York Academy of Sciences, v. 653, p. 202-205, 1992. 
BANCROFT, J.D.; STEVENS, A. Theory and Practice of Histological Techniques. New York: Churchill Livingstone Inc., 1996. 766 p.

BYRD, E.E.; DENTON, J.F. The helminth parasites of birds. I. A review of the trematode genus Tanaisia Skrjabin, 1924. American Midland Naturalist, v. 43, n. 1, p. 32-57, 1950.

De CARLI, G.A. Diagnóstico laboratorial das parasitoses humanas. Métodos e Técnicas. Rio de Janeiro: MEDSI Editora Médica e Científica Ltda., 1994. 315 p.

DOLLFUS, R.P. Sur un distome du genre Tamerlania K. I. Skrjabin 1924 avec un catalogue des trématodes des reins d'oiseaux. Annales de Parasitologie Humaine et Comparée, v. 21, n.1-2, p. 25-73, 1946.

FEDYNICH, A.M.; PENCE, D.B.; BERGAN, J.F. Helminth community structure and pattern in sympatric populations of black-bellied and fulvous whistling ducks. Canadian Journal of Zoology, v. 74, n. 12, p. 2219-2225, 1996.

FREITAS, J.F.T. Revisão da família Eucotylidae Skrjabin, 1924 (Trematoda). Memórias do Instituto Oswaldo Cruz, v. 49, n. 1, p. 33-123, 1951.

GOMES, D.C.; MENEZES, R.C.; TORTELLY, R.; PINTO, R.M. Pathology and first occurrence of the kidney trematode Paratanaisia bragai (Santos, 1934) Freitas, 1959 (Digenea: Eucotylidae) in Phasianus colchicus L., 1758, from Brazil. Memórias do Instituto Oswaldo Cruz, v. 100, n. 3, p. 285-288, 2005.

KANEV, I.; RADEV, V.; FRIED, B. Family Eucotylidae Cohn, 1904. In: GIBSON, D. J., BRAY, R. A.; JONES, A. (Eds). Keys to the Trematoda. Wallingford: CAB International, 2002. p. 147-152.
KELLER, D.G.; ARAÚJO, J.L.B. Ciclo evolutivo de Paratanaisia bragai (Santos, 1934) (Trematoda, Eucotylidae) com novo hospedeiro intermediário no Brasil: Leptinaria unilamellata (D’Orbigny, 1835) (Gastropoda, Pulmonata, Subulinidae) em condições de laboratório. Revista Brasileira de Parasitologia Veterinária, v. 1, n. 2, p. 89-90, 1992.

MALDONADO, J.F. The life cycle of Tamerlania bragai Santos, 1934 (Eucotylidae), a kidney fluke of domestic pigeons. Journal of Parasitology, v. 31, p. 306-314, 1945.

MALDONADO, J.F.; HOFFMAN, W.A. Tamerlania bragai, a parasite of pigeons in Puerto Rico. Journal of Parasitology, v. 27, p. 91, 1941.

ODENING, K. Zwei neue nierentrematoden der unterordnung Eucotylata (Digenea, Sporocystoinei) aus singvögeln Brasiliens und Vietnams. Zeitschrift für Parasitenkunde, v. 23, p. 491-503, 1963.

SANTOS, V. Monostome renal das aves domésticas. Revista do Departamento Nacional da Produção Animal, v. 1, n. 1, p. 203-215, 1934.

STUNKARD, H.W. The morphology of Tamerlania bragai dos Santos, 1934. Journal of Parasitology, v. 31, n. 5, p. 301-305, 1945.

TUBANGUI, M.A.; MASILUNGAN, V.A. Trematode parasites of Philippine Vertebrates. IX Flukes from the domestic fowl and other birds. Philippine Journal of Science, v. 75, n. 2, p. 131-141, 1941.

YAMAGUTI, S. Systema Helminthum. The Digenetic Trematodes of Vertebrates. New York: Interscience Publishers, 1958. v. 1, 979p.

Recebido em 14 de setembro de 2006.

Aceito para publicação em 06 de agosto de 2007. 